\title{
Pion scattering poles and chiral symmetry restoration
}

\author{
D. Fernández-Fraile* and A. Gómez Nicola ${ }^{\dagger}$ \\ Departamentos de Física Teórica I y II. Univ. Complutense. 28040 Madrid. Spain. \\ E. T. Herruzo \\ Instituto de Microelectrónica de Madrid, CSIC, Isaac Newton 8, 28760 Tres Cantos, Madrid, Spain
}

(Received 10 July 2007; published 24 October 2007)

\begin{abstract}
Using unitarized chiral perturbation theory methods, we perform a detailed analysis of the $\pi \pi$ scattering poles $f_{0}(600)$ and $\rho(770)$ behavior when medium effects such as temperature or density drive the system towards chiral symmetry restoration. In the analysis of real poles below threshold, we show that it is crucial to extend properly the unitarized amplitudes so that they match the perturbative Adler zeros. Our results do not show threshold enhancement effects at finite temperature in the $f_{0}(600)$ channel, which remains as a pole of broad nature. We also implement $T=0$ finite-density effects related to chiral symmetry restoration, by varying the pole position with the pion decay constant. Although this approach takes into account only a limited class of contributions, we reproduce the expected finite-density restoration behavior, which drives the poles towards the real axis, producing threshold enhancement and $\pi \pi$ bound states. We compare our results with several model approaches and discuss the experimental consequences, both in relativistic heavy ion collisions and in $\pi \rightarrow \pi \pi$ and $\gamma \rightarrow \pi \pi$ reactions in nuclei.
\end{abstract}

DOI: 10.1103/PhysRevD.76.085020

PACS numbers: 11.10.Wx, 12.39.Fe, 13.75.Lb

\section{INTRODUCTION}

Chiral symmetry restoration in a hot and dense environment and its possible experimental signatures are central issues in the present program of relativistic heavy ion collisions and reactions in nuclear matter. In principle, it should manifest both in the vanishing of the chiral condensate and in the degeneracy of the possible chiral partners. An important role in this context is played by the $\sigma$, the $I=J=0$ state corresponding physically to the $f_{0}(600)$ broad resonance listed by the Particle Data Group [1] and observed as a pole in $\pi \pi$ scattering. Since this state shares the vacuum quantum numbers, it is a suitable channel to study possible medium effects that may hint towards chiral symmetry restoration. An interesting suggestion in this context is the formation of a very narrow state near the two-pion threshold as a precursor of the chiral transition. This was first suggested in [2] in a NJL model calculation and later on also in the $O(4)$ model at finite temperature [3]. The presence of such a state was explained in the following way. The mass of the $\sigma$ is reduced from its vacuum value towards the mass of the pion by medium effects restoring the symmetry. As $M_{\sigma}$ gets closer to the pion threshold $2 m_{\pi}$ (which also changes with medium effects) the phase space of the dominant decay $\sigma \rightarrow \pi \pi$ is squeezed, so that the decay width goes to zero and the physical $\sigma$ pole approaches the real axis. As a consequence, there is a threshold enhancement of the $\sigma$ spectral function [3]. Such an enhancement is produced both by finite temperature and by nuclear density effects

\footnotetext{
*danfer@fis.ucm.es

gomez@fis.ucm.es

Elena.Tomas@imm.cnm.csic.es
}

[4] and should, in principle, imply a strong enhancement of the $\pi \pi$ cross section in the $I=J=0$ channel $[5,6]$. The analysis of the $\pi \pi$ scattering amplitude in the NJL model shows analogous results at finite density [7].

More recent analyses have shown some important differences with the works by Hatsuda and collaborators discussed above, especially in the finite- $T$ case. A large $N$ analysis in the $O(N)$ model [8] shows that the physical $\sigma$ pole in the second Riemann sheet has still a sizable width at the temperature for which the real part of the pole has already reached $2 m_{\pi}$. Further increasing of medium effects does reduce the imaginary part to zero. At that moment, two poles coexist on the real axis: one of them is the original second-sheet $\sigma$ pole and the other one comes from its unphysical partner in the upper half plane. From there on, one of the poles moves onto the first Riemann sheet, becoming a stable bound state. In fact, there had been earlier proposals about the formation of a $\pi \pi$ bound state in nuclear matter as a consequence of the increasing in-medium strength [9]. This behavior is argued in [10] to be universal, provided there is a stable state above the critical point. In $[11,12]$ a self-energy study of the $\sigma$ pole at finite $T$ in the $O(4)$ model is also presented, but the results are slightly different from [8]. These authors find a real pole in the second Riemann sheet for all temperatures, coexisting with the physical $\sigma$ pole. The real pole moves with $T$ towards threshold, causing threshold enhancement when it arrives $2 m_{\pi}$. However, the enhancement is smeared out if the pion thermal width is taken into account [11]. From that temperature onwards, that pole crosses to the first sheet, becoming a finite temperature $\pi \pi$ bound state, while the physical $\sigma$ pole remains on the second sheet, reducing its real part towards $2 m_{\pi}$ but keeping a nonzero imaginary part and its broad nature. Finally, it is worth 
mentioning also the analysis in $[13,14]$ of nuclear effects in $\pi \pi$ scattering using effective Lagrangians in a chiral unitary approach, where a weaker threshold effect than in [5] is obtained, and the importance of $p$-wave pion renormalization in $\pi \pi$ scattering is stressed.

Experimentally, there is evidence for a $I=J=0 \pi \pi$ threshold effect induced by the nuclear medium in $\pi A \rightarrow$ $\pi \pi A^{\prime}[15,16]$ and $\gamma A \rightarrow \pi \pi A^{\prime}$ [17] reactions in nuclei. The CHAOS collaboration [15] has reported a much stronger effect than the Crystal-Ball one [16]. There is then some controversy both in the results and in their interpretation. Thus, while in [15] a peak in the two-pion invariant mass distribution is seen at threshold for increasing nuclear density, the results being compatible both with chiral symmetry restoration and ordinary nuclear effects in the $\sigma$ channel, the effect in $[16,17]$ is weaker and seems to be better described by models in which the $\sigma$ appears as a broad $\pi \pi$ resonance [13]. No such threshold nuclear effect is seen in the isospin channels $I=2$ [15] and $I=1$ [17].

In this work we will use unitarized chiral perturbation theory (ChPT) to investigate the behavior of the pion scattering poles as the system approaches chiral symmetry restoration, with the sole physical input of chiral symmetry and unitarity. Although ChPT provides a perturbative expansion in meson energies and temperatures and therefore it is not capable of predicting a true chiral phase transition, its predictions are based only on the chiral symmetry breaking pattern and are therefore model independent. In fact, the quark condensate calculated within ChPT shows a restoring behavior, predicting a critical temperature around $T_{c} \sim 200-250 \mathrm{MeV}$ when it is extrapolated from the low- $T$ region [18-20]. On the other hand, the requirement of exact unitarity within the ChPT framework has led to a fruitful unitarization program of the meson-meson scattering amplitudes [21-24], one of its main results being the generation of all the low-lying meson resonances with their masses and widths in agreement with the values quoted by the PDG [1]. Recently, the pion scattering amplitudes have been calculated and unitarized at finite temperature $[25,26]$, which has allowed us to determine the low- $T$ evolution of the $f_{0}(600)$ and $\rho(770)$ poles. Here, we will study those poles, attending to their relation with chiral symmetry restoration. The specific new aspects that will be studied here and were not considered before are: (i) Precursors of chiral symmetry restoration such as threshold enhancement, emphasizing the modifications of the original arguments when a not-narrow state is present (Sec. III), (ii) A detailed analysis of poles in the real axis below the $\pi \pi$ threshold, both in the second (virtual states) and in the first (bound states) Riemann sheets (Sec. IV). This will require a generalization of the unitarized amplitudes in order to account properly for the presence of Adler zeros and remove unphysical poles, which is also a new result in vacuum. (iii) The nature of the $f_{0}(600)$ and $\rho$ states as revealed by their thermal and in-medium behavior
(Secs. V and VI) and (iv) The analysis of nuclear density chiral restoring effects (Sec. VI).

\section{FORMALISM}

Chiral perturbation theory is the most general lowenergy framework compatible with the $S U_{L}\left(N_{f}\right) \times$ $S U_{R}\left(N_{f}\right) \rightarrow S U_{V}\left(N_{f}\right) \quad$ Spontaneous chiral symmetry breaking pattern of QCD with $N_{f}=2,3$ light quark flavors. We will restrict to the two flavor case here. ChPT is built as an expansion in $p / \Lambda_{\chi}, p$ denoting generically any pion momenta or mass and $\Lambda_{\chi} \simeq 4 \pi f_{\pi} \simeq 1 \mathrm{GeV}$ is the typical chiral scale. Considering the heat bath temperature $T$ as a formally $\mathcal{O}(p)$ quantity, ChPT can also be used to study a meson gas at temperatures below $T_{c}[18,19]$.

The original calculation of pion-pion elastic scattering amplitudes in ChPT to one loop was given in [27] and its finite- $T$ extension is discussed in [25]. Consider a partial wave $t^{I J}(s ; T)$ with definite isospin $I$ and angular momentum $J$, where $s$ is the center of mass energy squared of the pion pair. The generic ChPT structure is then $t^{I J}(s ; T)=$ $t_{2}^{I J}(s)+t_{4}^{I J}(s ; T)+\ldots$, where $t_{k}$ denotes the $\mathcal{O}\left(p / \Lambda_{\chi}\right)^{k}$ contribution. The lowest order $t_{2}(s)$ is temperatureindependent and gives Weinberg's low-energy theorem [28] from the lowest order chiral Lagrangian $\mathcal{L}_{2}$ (the nonlinear sigma model). It has the following form:

$$
t_{2}^{I J}(s)=A^{I J}\left(s-s_{0}^{I J}\right) .
$$

Here, $s_{0}$ is the so called Adler zero (the point where the amplitude vanishes) to second order. For the relevant partial waves in pion scattering, the values of the $A$ and $s_{0}$ constants are given in Table I. Note that to this order, the amplitudes depend only on the pion mass $m_{\pi} \simeq 140 \mathrm{MeV}$ and the pion decay constant $f_{\pi} \simeq 93 \mathrm{MeV}$. The $t_{4}$ term includes contributions both from tree level $\mathcal{L}_{4}$ and oneloop $\mathcal{L}_{2}$ graphs. The tree level contributions are $T$-independent, renormalize the amplitudes to this order and depend on certain low-energy constants. We follow the convention in [27] and write the amplitude in terms of the $\bar{l}_{i}$ constants, which are independent of the renormalization scale. The one-loop pion scattering amplitude depends only on $\bar{l}_{1}$ and $\bar{l}_{2}$, but if it is written in terms of the physical $m_{\pi}$ and $f_{\pi}$, as we do here, then it also depends on $\bar{l}_{3}$ and $\bar{l}_{4}$. The numerical values of the low-energy constants are fixed phenomenologically. We will use the same values as in [26], namely $\bar{l}_{1}=-0.3, \bar{l}_{2}=5.6, \bar{l}_{3}=3.4$ and $\bar{l}_{4}=4.3$,

TABLE I. Values of the constants for the partial waves at lowest order in ChPT.

\begin{tabular}{lcc}
\hline \hline$I J$ & $16 \pi f_{\pi}^{2} A^{I J}$ & $s_{0}^{I J} / m_{\pi}^{2}$ \\
\hline 00 & 1 & $1 / 2$ \\
11 & $1 / 6$ & 4 \\
20 & $-1 / 2$ & 2 \\
\hline \hline
\end{tabular}


which fit the mass and width of the $\rho(770)$ (see below) when the partial waves are unitarized.

The one-loop part in $t_{4}$ contains, in particular, the imaginary part required by unitarity. At $T=0$, exact unitarity for partial waves reads $\operatorname{Im} t^{I J}(s)=\sigma_{0}(s)\left|t^{I J}(s)\right|^{2}$ or, for the inverse amplitude:

$$
\operatorname{Im}\left[\frac{1}{t^{I J}(s)}\right]=-\sigma_{0}(s) \Rightarrow t^{I J}(s)=\frac{1}{\operatorname{Re}\left[1 / t^{I J}(s)\right]-i \sigma_{0}}
$$

above the two-pion threshold, i.e., for $s>4 m_{\pi}^{2}$ where $\sigma_{0}(s)=\sqrt{1-4 m_{\pi}^{2} / s}$ is the two-pion phase space. The ChPT expansion cannot reproduce unitarity exactly, but only perturbatively, i.e., $\operatorname{Im} t_{4}=\sigma_{0}(s)\left|t_{2}(s)\right|^{2}$ and so on. Typically, the unitarity violations are more severe as energy increases, especially if there are physical resonances with the same quantum numbers as the channel under consideration. For these reasons, several unitarization approaches have been proposed, which amount to approximate $\operatorname{Re}[1 / t]$ in (2) in different ways. A simple and successful one within the ChPT context is the so-called inverse amplitude method (IAM) [21,22] which is built by demanding exact unitarity and matching with the ChPT expansion up to fourth order when expanded at low energies, i.e., $t^{\mathrm{IAM}}=t_{2}+t_{4}+\ldots$, where the different chiral orders can be traced by counting powers of $1 / f_{\pi}^{2}$. We recall that the perturbative expansion is meant to converge at $T=$ 0 near threshold so that it is in that region where the formal expansion in inverse powers of $f_{\pi}^{2}$ has a physical meaning. With these two requirements, the IAM amplitude reads for a given partial wave $t^{\mathrm{IAM}}=\left[t_{2}\right]^{2} /\left(t_{2}-t_{4}\right)$.

At finite $T$, the partial waves calculated in [25] satisfy the following thermal perturbative unitarity relation:

$$
\operatorname{Im} t_{4}(s ; T)=\sigma_{T}(s)\left|t_{2}(s)\right|^{2}
$$

where

$$
\sigma_{T}(s)=\sigma_{0}(s)\left[1+2 n_{B}(\sqrt{s} / 2)\right]
$$

and $n_{B}(x)=(\exp (x / T)-1)^{-1}$ is the Bose-Einstein distribution function.

The function $\sigma_{T}(s)$ is the thermal phase space [29], which is increased with respect to the $T=0$ one by the difference $\left[1+n_{B}\left(E_{1}\right)\right]\left[1+n_{B}\left(E_{2}\right)\right]-n_{B}\left(E_{1}\right) n_{B}\left(E_{2}\right)=$ $1+n_{B}\left(E_{1}\right)+n_{B}\left(E_{2}\right)$, where $E_{1,2}$ are the energies of the two colliding pions. The first term in the difference corresponds to the enhancement of the available phase space due to the increase of two-pion states by the scattering of two pions in the thermal bath (these are the stimulated emission processes discussed in [29]). The second term reduces the phase space by absorption due to collisions of the incoming pions with the thermal bath ones. In the center of mass frame, $E_{1}=E_{2}=\sqrt{s} / 2$ and the thermal phase space reduces to (4). Therefore, one can extend the usual $T=0$ exact unitarity arguments, replacing $\sigma_{0} \rightarrow \sigma_{T}$ and the partial waves by the finite- $T$ ones, provided that only intermediate two-pion states are relevant in the ther- mal bath. This is reasonable if we remain in a dilute gas regime, which is consistent at low and moderate temperatures. Thus, from the perturbative amplitude in the center of mass frame, following the same steps as for $T=0$ case, the unitarized IAM thermal partial waves for every $I J$ channel are given by [26]:

$$
t^{\mathrm{IAM}}(s ; T)=\frac{\left[t_{2}(s)\right]^{2}}{t_{2}(s)-t_{4}(s ; T)} .
$$

Therefore, the above thermal unitarized amplitude satisfies the exact unitarity relation (2) with $\sigma_{0}$ replaced by $\sigma_{T}$ and the partial waves replaced by their finite- $T$ counterparts obtained in [25]. As a consequence, it has the same analytic structure as the $T=0$ one, i.e., it is analytical on the complex plane off the real axis, it has the right unitarity cut starting at the two-pion threshold $s=4 m_{\pi}^{2}$ and a left cut for $s<0$ coming from diagrams in the $t-u$ channels [26]. From these analytical properties, one can immediately define the amplitude on the second Riemann sheet which continuously connects with the imaginary part of the first Riemann sheet amplitude $t^{\mathrm{IAM}}$ in (5) across the right cut, i.e., $\quad \operatorname{Im} t^{I I}(s-i \epsilon)=\operatorname{Im} t^{I}(s+i \epsilon)=\sigma_{T}(s)\left|t^{I}\right|^{2}=$ $-\operatorname{Im} t^{I}(s-i \epsilon)$ for $s>4 m_{\pi}^{2}$. One has:

$$
t^{I I}(s ; T)=\frac{t^{I}(s ; T)}{1-2 i \sigma_{T}(s) t^{I}(s ; T)}
$$

The poles of $t^{I I}$ in the lower complex half plane correspond to physical resonances. We follow the standard convention and denote the pole position by $s_{\text {pole }}=\left(M_{p}-\right.$ $\left.i \Gamma_{p} / 2\right)^{2}$ [1] chosen so that $M_{p}$ and $\Gamma_{p}$ would correspond to the mass and width of a narrow Breit-Wigner resonance. Since we are working by definition in the center of mass frame of the incoming pions, the masses and widths correspond to resonances at rest. At $T=0$, and with the lowenergy constants chosen above, $t^{00, I I}$ has a pole at $M_{p} \simeq$ $441 \mathrm{MeV}$ and $\Gamma_{p} \simeq 464 \mathrm{MeV}$, corresponding to the $f_{0}(600)$. Note that this state is clearly not a simple narrow Breit-Wigner resonance, since its width is of the same size as its mass. In the $I=J=1$ channel, the pole is at $M_{p} \simeq$ $756 \mathrm{MeV}, \Gamma_{p} \simeq 151 \mathrm{MeV}$, corresponding to the $\rho(770)$ [1].

\section{THERMAL EVOLUTION OF THE POLES}

At $T \neq 0$, the IAM poles are shown in Fig. 1 . The $\rho$ pole follows a finite- $T$ trajectory compatible with dilepton data in relativistic heavy ion collisions, namely, a broadening behavior with only a small mass decreasing. This point will be commented in more detail in Sec. V. The thermal widening in the $\rho$ channel can be explained in part due to the increase of thermal phase space given by $\sigma_{T}$. In fact, it can be shown [26] that if the change in the mass and in the effective $\rho \pi \pi$ coupling constant are neglected (see below) the width would increase simply as $\Gamma_{T} / \Gamma_{0}=$ $\sigma_{T}\left(M_{\rho}^{2}\right) / \sigma_{0}\left(M_{\rho}^{2}\right)=\left[1+2 n_{B}\left(M_{\rho} / 2\right)\right]$, which is rather ac- 

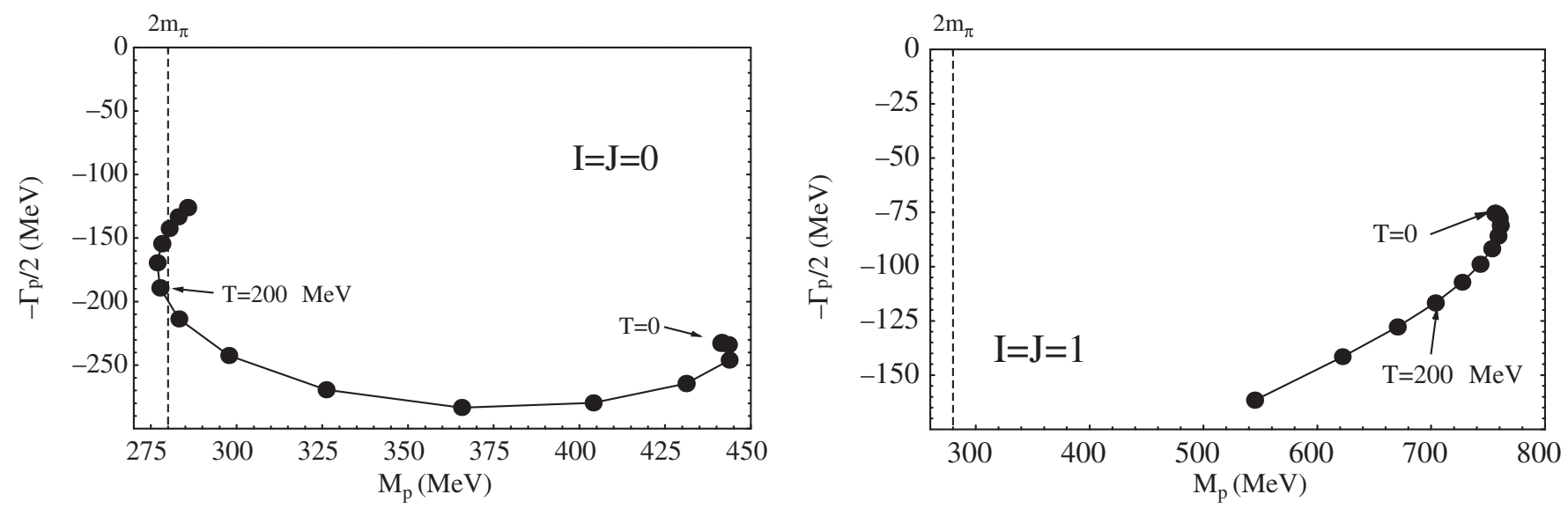

FIG. 1. Evolution with temperature of the unitarized $f_{0}(600)$ and $\rho$ complex poles in the second Riemann sheet. The points are obtained by varying the temperature in $20 \mathrm{MeV}$ intervals.

curate up to $T \simeq 100 \mathrm{MeV}$. For higher $T$, there is a further increase of the width due to the increase of the effective vertex [26].

The phase space argument cannot be directly applied to the $f_{0}(600)$ pole, since this is a broad resonance. Still, there is a broadening effect for moderate temperatures, as it can be seen in Fig. 1. However, this behavior changes qualitatively when the temperature is further increased, due to the effect of chiral symmetry restoration, which is notorious in this channel. The system tends to restore chiral symmetry by reducing drastically the mass and in this sense this state tends to become degenerate with the chiral pion triplet (see Sec. V). In principle, the argument proposed in [2,3] would suggest that as $M_{p} \rightarrow 2 m_{\pi}$, the width should go to zero by phase space reduction. However, it is very important to note that this is a narrow resonance argument and it does not necessarily hold for a wide state, as it is the case here. In fact, observe that our pole remains as a wide state even at temperatures close to the phase transition, where its mass has already reached the two-pion threshold. In fact, due to the initial phase space widening, the width close to the transition is of the same order as for $T=0$. This is in agreement with the behavior found in [8] below the transition and it is one of our relevant results. Thus, we do not expect this pole to cause any sizable effect in the real axis near threshold, like an enhancement of the cross section, which could be regarded as a precursor of chiral symmetry restoration. This is indeed the case, as observed in Fig. 2. Note that almost no variation with temperature is seen at threshold, i.e., the scattering length remains almost $T$-independent. This result had also been obtained in the finite- $T$ scattering length calculation in [30] and a qualitative explanation is provided below. To fully confirm the absence of chiral restoration precursors at finite $T$, we need to perform an analysis of the real poles, which deserve a separate treatment for the reasons explained in Sec. IV.

Let us now provide a qualitative description of the above results. Consider the propagator and spectral function of the resonance under consideration at rest:

$$
\begin{gathered}
D(s)=\frac{1}{s-M^{2}(s)+i M(s) \Gamma(s)} \\
\rho(s)=-2 \operatorname{Im} D=\frac{2 M(s) \Gamma(s)}{\left[s-M^{2}(s)\right]^{2}+[M(s) \Gamma(s)]^{2}} .
\end{gathered}
$$

Recall that (8) reduces to $\rho=2 \pi \delta\left(s-M^{2}\right)$ when $\Gamma \rightarrow$ $0^{+}$, as it corresponds to a free particle of mass $M$ and positive energy. Here we are denoting by $M^{2}(s)$ and $[-M(s) \Gamma(s)]$ the real and imaginary parts of the resonance self-energy, respectively, with both $M$ and $\Gamma$ real functions of $s$ in the general case. In the complex $s$ plane, the pole of the propagator is the solution of $s_{\text {pole }}=M^{2}\left(s_{\text {pole }}\right)-$ $i M\left(s_{\text {pole }}\right) \Gamma\left(s_{\text {pole }}\right)$. Thus, with our convention for the poles, we have

$$
M^{2}\left(s_{\text {pole }}\right)=M_{p}^{2}-\frac{\Gamma_{p}^{2}}{4} ; \quad \Gamma^{2}\left(s_{\text {pole }}\right)=\frac{M_{p}^{2} \Gamma_{p}^{2}}{M_{p}^{2}-\Gamma_{p}^{2} / 4} .
$$

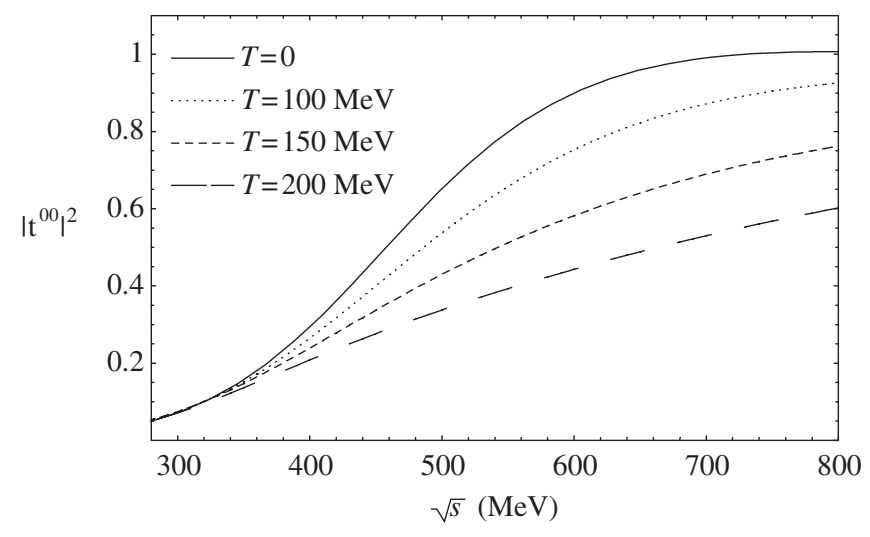

FIG. 2. Squared modulus of the $t^{00}$ unitarized partial wave for different temperatures. 
Consider now the contribution to the $\pi \pi$ scattering amplitude of the exchange of a resonance $R$ with propagator (7) and the same quantum numbers as the corresponding $I J$ channel:

$$
t^{e x}(s)=\frac{-M(s) \alpha(s)}{s-M^{2}(s)+i M(s) \Gamma(s)}
$$

where $M \alpha$ is the effective $\pi \pi R$ vertex squared. The parametrization (10) of the partial waves can be made unitary by demanding (2) in (10), which gives $\Gamma(s)=\sigma_{0}(s) \alpha(s)$ for $s \in \mathbb{R}$ and $s>4 m_{\pi}^{2}$. This parametrization is therefore compatible with chiral symmetry and unitarity, although a correct description of the 00 channel in the real axis would also need the addition of background contributions [31], which does not change our qualitative arguments here. As far as we do not make any claim about the relative size of $\Gamma$ and $M$ nor about their behavior with $s$, the parametrization (10) can be viewed as a generalized Breit-Wigner form on the real axis above threshold.

It is instructive at this point to remind the narrow resonance limit behavior. If $\Gamma_{p} \ll M_{p}$, the pole is close to the real axis, so that the amplitude on the real axis is peaked around $s=M^{2} \simeq M_{p}^{2}$ and the width $\Gamma_{p} \simeq \Gamma\left(M^{2}\right)=$ $\alpha\left(M^{2}\right) \sigma_{0}\left(M^{2}\right) \theta\left(M^{2}-4 m_{\pi}^{2}\right)$. If $M \rightarrow 2 m_{\pi}$, driven for instance by chiral restoration in the 00 channel, the spectral function (8) and $\operatorname{Im} t$ are then enhanced for $s$ near threshold as

$$
\begin{gathered}
\rho(s) \sim \frac{\theta\left(s-4 m_{\pi}^{2}\right)}{\alpha \sqrt{s-4 m_{\pi}^{2}}} \\
\operatorname{Im} t(s) \sim \frac{2 m_{\pi} \theta\left(s-4 m_{\pi}^{2}\right)}{\sqrt{s-4 m_{\pi}^{2}}} .
\end{gathered}
$$

This is the typical threshold enhancement produced by phase space squeezing $[3,4]$ which consequently implies an enhancement in the $\pi \pi$ cross section, as explained before. On the other hand, if medium effects do not change $M$ and $\alpha$ much, as it happens for the $\rho$, the only temperature modification is the replacement of the phase space $\sigma_{0} \rightarrow$ $\sigma_{T}$, which leads to $\Gamma_{T} / \Gamma_{0}=\sigma_{T} / \sigma_{0}$, as announced above.

Let us now show how the previous arguments change when the resonant state is not narrow, i.e., when the pole position values $\Gamma_{p}$ and $M_{p}$ are of the same order. For that purpose, we will consider, as a working example, a BWlike form (10) for the amplitude.

First, in the broad resonance case, the dependence of the functions $M, \alpha, \Gamma$ with $s$ may be important. For instance, if $M$ is roughly constant and large compared to $\Gamma$, a formal $s$-expansion of (10) gives $t \sim \alpha / M$. Comparing this to the lowest ChPT order $t_{2}$ in (1) (required by chiral symmetry) would give $\alpha \sim A M\left(s-s_{0}\right)$ near threshold. If $s_{0} \neq 4 m_{\pi}^{2}$ as in the 00 channel, that behavior for $\alpha$ is compatible with the enhancement as $M \rightarrow 2 m_{\pi}$, since near threshold the denominator of (10) is dominated by its imaginary part $\Gamma \sim \sigma_{0}$ as compared with the real part $\left(s-M^{2}\right) \sim \sigma_{0}^{2}$.
However, this need not be the case if $M$ as given by (9) is not large compared to $2 m_{\pi}$ [for the $f_{0}(600), M \sim$ $373.3 \mathrm{MeV}$ already at $T=0$ and it gets further reduced at finite $T$ ]. In that case, $t(s)$ in (10) near threshold can be made compatible with $t_{2}$ by taking $\alpha \sim-A\left(s-s_{0}\right)(s-$ $\left.M^{2}\right) / M$, so that $t(s) \sim(-M \alpha) /\left(s-M^{2}\right) \sim A\left(s-s_{0}\right)$ near threshold, even if $M \rightarrow 2 m_{\pi}$ at a certain temperature, since now $\Gamma \sim \sigma_{0}^{3}$ so that the $\sigma_{0}^{2}$ in the real part of the denominator dominates. The result is now that $t(s)$ near threshold is roughly independent of $M$ and therefore of $T$, with no threshold enhancement. This is consistent with what we see in Fig. 2, which means that the leading order ChPT expression ( $T$-independent) basically dominates at all temperatures near threshold.

Second, the phase space vanishing of the width near threshold for narrow resonances relies heavily on the fact that the spectral function is peaked around $s \sim M^{2}$ or, in other words, that the pole is reasonably close to the real axis. However, for the $f_{0}(600)$, the spectral function is broadly distributed so that the phase space $\sigma_{T}(s)$ is not directly evaluated at $s=M^{2}$. An explicit way to see this is to consider the decay rate of the $R \rightarrow \pi \pi$ process. In the narrow resonance limit, $R$ can be considered a particle with four-momentum $P^{2}=M_{R}^{2}$ so that the differential decay rate in the rest frame is given by

$$
d \Gamma_{D}=\frac{1}{2} \frac{1}{2 M_{R}}|\langle R|T| \pi \pi\rangle|^{2} d \Phi_{12}
$$

where

$$
d \Phi_{12}=\prod_{i=1,2} \frac{d^{3} \vec{p}_{i}}{(2 \pi)^{3} 2 E_{i}}(2 \pi)^{4} \delta^{(4)}\left[P-p_{1}-p_{2}\right]
$$

is the two-particle differential phase space, $P=\left(M_{R}, \overrightarrow{0}\right)$, $E_{i}^{2}=\left|\vec{p}_{i}\right|^{2}+m_{\pi}^{2}$ and therefore $s=\left(p_{1}+p_{2}\right)^{2}=P^{2}=M_{R}^{2}$. Replacing the $R \rightarrow \pi \pi T$-matrix element in (13) by $M \alpha$, which is assumed to depend only on the energy of the $R$ state, and integrating the phase space $\int d \Phi_{12}=\sigma_{0}(s) \theta(s-$ $\left.4 m_{\pi}^{2}\right) /(8 \pi)$ gives for the decay rate $\Gamma_{D}=\sigma_{0}\left(M^{2}\right) \times$ $\alpha\left(M^{2}\right) \theta\left(s-4 m_{\pi}^{2}\right) /(32 \pi)=\Gamma\left(M^{2}\right) /(32 \pi) \simeq \Gamma_{p} /(32 \pi)$, yielding the standard relation between the width and the decay rate, which is also suppressed by phase space when the mass of the state approaches threshold.

If the spectral function of the resonance is not well approximated by a $\delta$ function, the differential decay width (13) is generalized by replacing $\frac{1}{2 M_{R}} \rightarrow \int(d \omega / 2 \pi) \times$ $\rho(\omega) \theta(\omega)$ so that instead of $\Gamma_{D}$ one has the number of decays per unit volume and per unit of four-momenta [32], integrated over the energy $\omega$ distributed according to the spectral function of the resonant state. Accordingly, with the spectral function (8) and replacing $M, \Gamma, \sigma_{0}, \alpha$ by their $T$-dependent counterparts, the quantity 


$$
\begin{aligned}
F_{T}= & \int_{4 m_{\pi}^{2}}^{\infty} \frac{d s}{2 \pi} \frac{M_{T}(s) \alpha_{T}(s) \sigma_{T}(s)}{2 \sqrt{s}} \\
& \times \frac{2 M_{T}(s) \Gamma_{T}(s)}{\left[s-M_{T}^{2}(s)\right]^{2}+\left[M_{T}(s) \Gamma_{T}(s)\right]^{2}}
\end{aligned}
$$

should behave qualitatively as a generalized decay rate and therefore we expect it to follow a similar pattern as the imaginary part of the pole position $\Gamma_{p}(T)$. As a consistency check, in the narrow resonance limit $F_{T}=\alpha_{T} \sigma_{T} \theta\left(M_{T}^{2}-\right.$ $4 m_{\pi}^{2}$ ). In the general case though, when $M_{T} \rightarrow 2 m_{\pi}$, there will be a nonvanishing contribution to the integral due to the broad spectral function.

Obviously, we cannot draw any conclusion about the evolution of $F_{T}$ without specifying the $T$ and $s$ dependence of $\alpha_{T}, M_{T}, \Gamma_{T}$. In order to check that this is a consistent description, let us choose for simplicity $s$-independent $M_{T}$ and $\Gamma_{T}$, related to the pole position values by (9), with the values of $\Gamma_{p}$ and $M_{p}$ obtained in our full IAM calculation. We also take $\alpha_{T}=\Gamma_{T}$, which is consistent with the unitarity requirement away from threshold (the spectral function in the integrand is suppressed for $s$ near threshold in the broad case, according to our previous arguments). We emphasize that this is a rough approximation, since the actual profile of the $t^{00}$ partial wave does not resemble a BW-like form, even with large $\Gamma$ [31]. Nevertheless, it provides us with a simple model to explain a thermal resonance behavior similar to the one we obtain. With that simplification, $F_{T}$ rises with $T$ until $T \sim 100 \mathrm{MeV}$ $\left(F_{100} / F_{0} \simeq 1.1\right)$ due to phase space increasing in the numerator of (15) and then starts decreasing as the real part of the pole approaches threshold, reaching a value $F_{200} / F_{0} \simeq$ 0.7. Comparing with the evolution of $\Gamma_{p}$ in Fig. 1, where $\Gamma_{p}(100 \mathrm{MeV}) / \Gamma_{p}(0) \simeq 1.2$ and $\Gamma_{p}(200 \mathrm{MeV}) / \Gamma_{p}(0) \simeq$ 0.8 , we see that a broad resonant thermal state with a finite generalized decay rate near the two-pion threshold behaves in a qualitatively similar way as our thermal $f_{0}(600)$ state.

From the above discussion we conclude that for a broad state like the $f_{0}(600)$ at finite temperature there is always a balance between thermal phase space increasing, which has nothing to do with chiral symmetry restoration, and chiral restoring mass and width decreasing. Depending on how broad the resonance is when chiral restoring effects start being important, the pole may move towards the real axis more or less rapidly. There is no way to know a priori whether it can be close enough to be considered a narrow resonance before the chiral transition, so that observable effects such as threshold enhancement may take place. In our approach, this does not happen at finite $T$ (the situation may change at finite density as we will see in Sec. VI) i.e., the $f_{0}(600)$ is still broad at the transition, in agreement with [8] but not with previous approaches [2,3]. We also agree with $[11,12]$ as far as the complex broad $I=J=0$ pole is concerned, and in the next section we will check for the presence of real poles.

\section{REAL AXIS POLES AND ADLER ZEROS}

The analysis of poles near the real axis below threshold is relevant for the chiral restoring behavior, as discussed above. As we will show here, the unitarized IAM amplitude below threshold has to be extended in order to account properly for the Adler zeros and remove spurious poles. For clarity, we will discuss the $T=0$ case first.

\section{A. $T=0$ extended amplitude}

Consider the IAM unitarized amplitude (5). Near threshold, $t^{\mathrm{IAM}} \sim t_{2}+t_{4}$ and $t_{4}$ is perturbatively small compared to $t_{2}$. On the other hand, the perturbative amplitude must have an Adler zero, i.e, a point where it vanishes, as a consequence of Weinberg's low-energy theorem [28], which predicts that the pion scattering amplitude vanishes in the chiral limit at $s=0$. Both $t_{2}$ and $t_{2}+t_{4}$ have this property and is natural to expect that $t^{\mathrm{IAM}}$ has a zero not far from the perturbative one. The zero of $t_{2}$ is given by $s_{0}$ in (1) and we will call $s_{1}$ the zero of $t_{2}+t_{4}$. Note that the IAM expression (5) vanishes exactly at $s_{0}$, although the order of the zero is not necessarily the same: if $t_{4}\left(s_{0}\right) \neq 0$, $t^{\mathrm{IAM}} \sim\left(s-s_{0}\right)^{2}$, while $t_{2}$ has a zero of order one. In addition, note that if $t_{2}$ and $t_{4}$ have the same sign and $t_{4}\left(s_{0}\right) \neq 0$, there is a point $\tilde{s}>s_{0}$ where the denominator of (5) vanishes. If that point exists for a given channel, it produces a spurious pole in the first Riemann sheet, since it would predict a not-observed $T=0 \pi \pi$ bound state. That pole does exist in the $I=J=0$ channel at $\tilde{s} \simeq(89 \mathrm{MeV})^{2}$ and at $\tilde{s} \simeq(197.4 \mathrm{MeV})^{2}$ for $I=2, J=0$. In the $I=J=$ 1 channel there is no such problem, since the scattering length has to vanish by parity so that $s_{0}=s_{1}=4 m_{\pi}^{2}$ and at that point both $t_{2}$ and $t_{4}$ vanish, so that (5) is not divergent. The position of $s_{0}, s_{1}$ and $\tilde{s}$ (all of them below threshold) for the 00 channel are shown in Fig. 3. The contribution of the possible amplitude zeros was already mentioned in early IAM papers [22], although it was not taken into

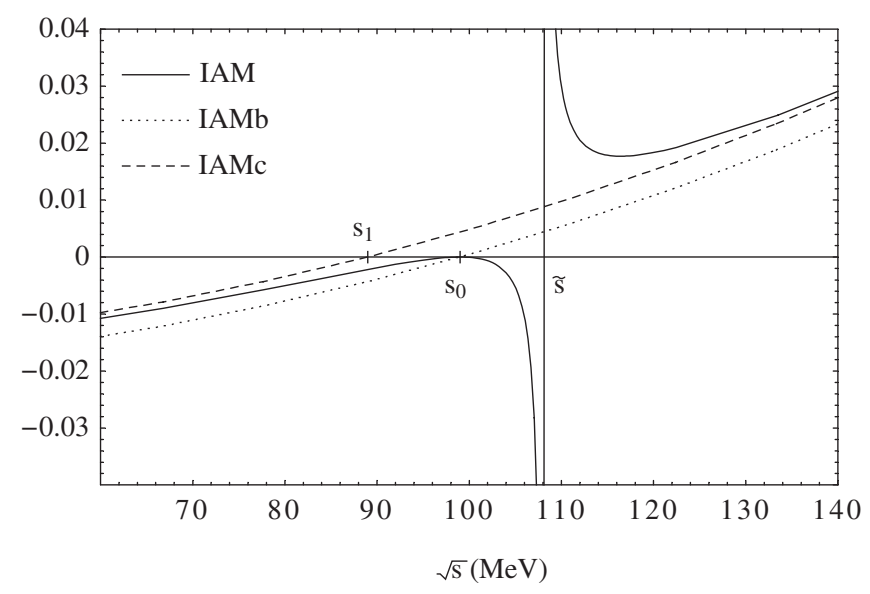

FIG. 3. Unitarized amplitudes at $T=0$ in the $I=J=0$ channel, where $s_{0}$ and $s_{1}$ are, respectively, the Adler zeros of second and fourth order and $\tilde{s}$ is the point where the IAM diverges. 
account since its effect is negligible in the physical region, as we will show below. The existence of the nonphysical pole has also been noticed in [33], where it is also linked to the presence of the Adler zero.

Therefore, the IAM definition (5) is affected with two related problems below threshold: the wrong order of the Adler zero and the presence of spurious poles. We will see now a simple way to cure both without spoiling the essential properties of chiral symmetry and unitarity of the IAM. For that purpose, we note that if we extend the inverse amplitude as:

$$
\frac{1}{t^{\mathrm{IAM}}(s)} \rightarrow \frac{1}{t^{\mathrm{IAM}}(s)}+g(s)
$$

with $g$ an analytic function off the real axis, real for $s \in \mathbb{R}$, the unitarity condition (2) remains unaltered and so does the analytic structure of the amplitude. The choice of $g$ is in principle arbitrary. Therefore, since having a double zero in the amplitude means having a double pole in the inverse amplitude, if we choose $g_{b}(s)=-R /\left(s-s_{0}\right)^{2}$ with $R$ the residue of $1 / t$ at the double pole $s_{0}$, we get an amplitude with an Adler zero of order one at $s=s_{0}$. In fact, note that, taking into account (1), the expansion of the inverse amplitude near $s_{0}$ reads

$$
\frac{1}{t^{\mathrm{IAM}}(s)}=-\frac{t_{4}\left(s_{0}\right)}{A^{2}\left(s-s_{0}\right)^{2}}+\frac{A-t_{4}^{\prime}\left(s_{0}\right)}{A^{2}\left(s-s_{0}\right)}+\ldots
$$

where the dots stand for terms which do not diverge as $s \rightarrow$ $s_{0}$.

Therefore, $R=-t_{4}\left(s_{0}\right) / A^{2}$ and the extended amplitude with this choice of $g$ becomes simply:

$$
t^{\mathrm{IAM} b}(s)=\frac{\left[t_{2}(s)\right]^{2}}{t_{2}(s)-t_{4}(s)+t_{4}\left(s_{0}\right)} .
$$

The expression (18) has a simple Adler zero at $s=s_{0}$ as long as $t_{4}^{\prime}\left(s_{0}\right) \neq 0$. But in addition, if $t_{2}(s)-t_{4}(s)$ is an increasing function of $s$ from $s=0$ to threshold, the IAM pole disappears, since in that case, $s_{0}$ is the only point where the denominator of (18) vanishes. This is what happens in the 00 channel, as shown in Fig. 3.

We can improve further the unitarized amplitude, by demanding also that it matches the ChPT series near threshold. Note that this condition does not hold for (18), which can easily be seen by reexpanding it in powers of $f_{\pi}^{-2}$. Since $t_{4}\left(s_{0}\right)=\mathcal{O}\left(f_{\pi}^{-4}\right)$, we get $t(s) \simeq t_{2}(s)+t_{4}(s)-$ $t_{4}\left(s_{0}\right)$ instead of the $t_{2}+t_{4}$ of ChPT. That is, it only matches correctly the first $O\left(f_{\pi}^{-2}\right)$ order $t_{2}$. If we want the amplitude to match $t_{2}+t_{4}$, then, as we will show below, it is sufficient to demand that both have the Adler zero at the same point, i.e., at $s=s_{1}$. Therefore, in order that $1 / t$ has a simple pole at that point an according to (17) we must subtract now the $s_{0}$ pole and add the $s_{1}$ one, i.e., we choose

$$
g_{c}(s)=\frac{1}{A^{2}}\left[\frac{t_{4}\left(s_{0}\right)}{\left(s-s_{0}\right)^{2}}-\frac{A-t_{4}^{\prime}\left(s_{0}\right)}{s-s_{0}}+\frac{c}{s-s_{1}}\right]
$$

where $c$ is an undetermined constant that we will fix by demanding the perturbative matching with the ChPT series to fourth order. Adding (19) to $1 / t^{\mathrm{IAM}}$ we get

$$
t^{\mathrm{IAM} c}(s)=\frac{A^{2}\left(s-s_{0}\right)^{2}\left(s-s_{1}\right)}{c\left(s-s_{0}\right)^{2}-\left(s-s_{1}\right)\left[t_{4}(s)-t_{4}\left(s_{0}\right)-\left(s-s_{0}\right) t_{4}^{\prime}\left(s_{0}\right)\right]} .
$$

Let us expand the previous expression in powers of $f_{\pi}^{-2}$. Recall that $A=\mathcal{O}\left(f_{\pi}^{-2}\right), \quad t_{4}=\mathcal{O}\left(f_{\pi}^{-4}\right)$ and $s_{1}=$ $s_{0}+\mathcal{O}\left(f_{\pi}^{-2}\right)$, so that the leading order is $t^{\mathrm{IAM} c}=\left(A^{2} / c\right) \times$ $\left(s-s_{0}\right)$ and therefore $c=A+\mathcal{O}\left(f_{\pi}^{-4}\right)$. Expanding now the expression $t_{2}\left(s_{1}\right)+t_{4}\left(s_{1}\right)=0$ around $s_{0}$ we find

$$
s_{1}=s_{0}-t_{4}\left(s_{0}\right) / A+\mathcal{O}\left(f_{\pi}^{-4}\right) .
$$

Using this in the expansion of (20) to fourth order, we have $t^{\mathrm{IAM} c}(s)=t_{2}(s)+t_{4}(s)-\left(s-s_{0}\right)\left(c-A+t_{4}^{\prime}\left(s_{0}\right)\right)+$ $\mathcal{O}\left(f_{\pi}^{-6}\right)$. Therefore, by taking

$$
c=A-t_{4}^{\prime}\left(s_{0}\right)
$$

in (20), the unitarized amplitude matches the chiral expansion up to fourth order and has the Adler zero at the same position and with the same order as the perturbative amplitude. Note also that with $c$ in (22), (20) reduces to (18) when $s_{1} \rightarrow s_{0}$, as it should. In fact, since the difference between $s_{1}$ and $s_{0}$ is perturbatively small, we expect this extended amplitude to behave very similarly as the simplest version (18), and, in particular, to remove the spurious pole while being numerically closer to the original IAM amplitude. That is indeed the case, as it can clearly be seen in Fig. 3. The same situation takes place in the $I=2, J=$ 0 channel. The extended amplitudes are expected to differ little from $t^{\mathrm{IAM}}$ away from $s_{0}$ or $s_{1}$, since the $g_{b, c}$ functions vanish for $s \gg s_{0}, s_{1}$. For that reason, in Fig. 3 we have only shown the region around the spurious pole and the Adler zero. Away from that region, either in the real axis or in the complex plane, there is practically no difference between the IAM or its extended versions discussed here.

It is interesting to note that if we had not redefined the amplitude, there would be also a nonphysical pole in the second Riemann sheet just below the real axis and below threshold. The IAM in the second Riemann sheet for $T=0$ reads, from (5) and (6):

$$
t^{\mathrm{IAM}, I I}=\frac{t_{2}^{2}}{t_{2}-t_{4}-2 \tilde{\sigma} t_{2}^{2}}
$$

where we have defined $\tilde{\sigma}(s)=i \sigma_{0}\left(s-i 0^{+}\right)=$ $\sqrt{4 m_{\pi}^{2}} / s-1$ for $0<s<4 m_{\pi}^{2}$. Thus, the denominator of (23) is positive near threshold for an attractive channel (dominated by $t_{2} \geq 0$ ) like the 00 one and diverges to minus infinity as $s \rightarrow 0^{+}$, so that it must have an odd number of zeros. Since the denominator is not zero at $s=$ $s_{0}$, that means that there would be at least one such real pole for the IAM in the second sheet. Consider however the extended amplitude, for instance (18). Now, 


$$
t^{\mathrm{IAM} b, I I}=\frac{t_{2}^{2}}{t_{2}-t_{4}+t_{4}\left(s_{0}\right)-2 \tilde{\sigma} t_{2}^{2}},
$$

which is not necessarily singular below threshold since both the denominator and the numerator vanish at $s=s_{0}$. A similar argument holds for $t^{\mathrm{IAM} c, I I}$, whose denominator is now positive both at threshold (provided again that the $\mathcal{O}\left(f_{\pi}^{2}\right)$ terms dominate there) and at $s=0$. The conclusion is then that for the extended amplitudes, there are no real poles in the second Riemann sheet below threshold, or an even number of them. We have checked that there are no such real poles at $T=0$. It is unclear whether the real second-sheet pole found in [11,12] (for the $\sigma$ self-energy) is also spurious, in the sense discussed here.

Finally, we have checked that the $\rho(770)$ and $f_{0}(600)$ poles remain at the same place at $T=0$ with the second Riemann sheet extensions of the extended amplitudes, as expected from our previous arguments. We also note that the above extended amplitudes can also be obtained by using dispersion relations [34], as it was also done in early derivations of the IAM [22], which provides a formal justification of the results shown here.

\section{B. $\boldsymbol{T} \neq \boldsymbol{0}$ corrections}

Once we have extended the $T=0$ amplitude to ensure the correct behavior below threshold, we obtain the extended $T \neq 0$ amplitudes by including the thermal corrections in $t_{4}(s ; T)$. Therefore, the values of $s_{1}$ and $\tilde{s}$ become temperature dependent. With this extended amplitude, we do not find any spurious pole in the first Riemann sheet, as it happened for $T=0$, up to temperatures close to the phase transition, nor any additional pole in the second sheet apart from the standard $f_{0}(600)$ pole. The results are shown in Fig. 4 with the extended amplitude $t^{\mathrm{IAM} c}(s ; T)$. We remark that this is consistent with the fact that the $f_{0}(600)$ pole shown in Fig. 1 remains far from the real axis near the phase transition. Our result in this respect is different from that in $[11,12]$, where an additional pole in the second sheet is found for all temperatures, although we agree with [11] in that finite- $T$ threshold effect are not seen. We also agree with [8], where the additional pole does not appear for low and moderate $T$.

An important remark is that temperature effects make $t_{4}(s ; T)$ grow with respect to $t_{2}(s)$ so that some of our previous $T=0$ conclusions could change. For instance, regarding the number of poles, the denominator of (18) or (20) could become now negative at threshold, so that we would have an odd number of real poles in the second Riemann sheet and also in the first one, which would correspond to $\pi \pi$ bound states. Although this is not the case at finite temperature, we will see in Sec. VI that it is possible that other chirally restoring effects, such as nuclear density, may cause the appearance of new poles in both sheets, as those obtained for instance in [8] and consistently with threshold enhancement nuclear effects.

\section{THE NATURE OF THE THERMAL RESONANCES}

The analysis of $\pi \pi$ scattering poles at finite temperature or density may also be useful to understand the nature of the resonant states in terms of quark and gluon degrees of freedom. This is particularly relevant for the $f_{0}(600)$, for which there is a long-standing controversy about its nature. In $O(4)$ or linear- $\sigma$ models, the $\sigma$ is introduced as an explicit degree of freedom in the Lagrangian, and by definition it transforms as a pure $\bar{q} q$, isospin singlet state [35]. On the other hand, the $f_{0}(600)$ listed by the PDG [1] is described by several approaches, including unitarized ChPT [22-24], where resonant states are dynamically generated, i.e., not included as explicit degrees of freedom from the start, nor making any requirement about their nature. When strangeness is included, unitarized ChPT generates precisely the scalar resonances corresponding to a scalar nonet pattern $\left(f_{0}(600), f_{0}(980), a_{0}(980)\right.$ and the controversial $\kappa)$ as well as the standard vector meson

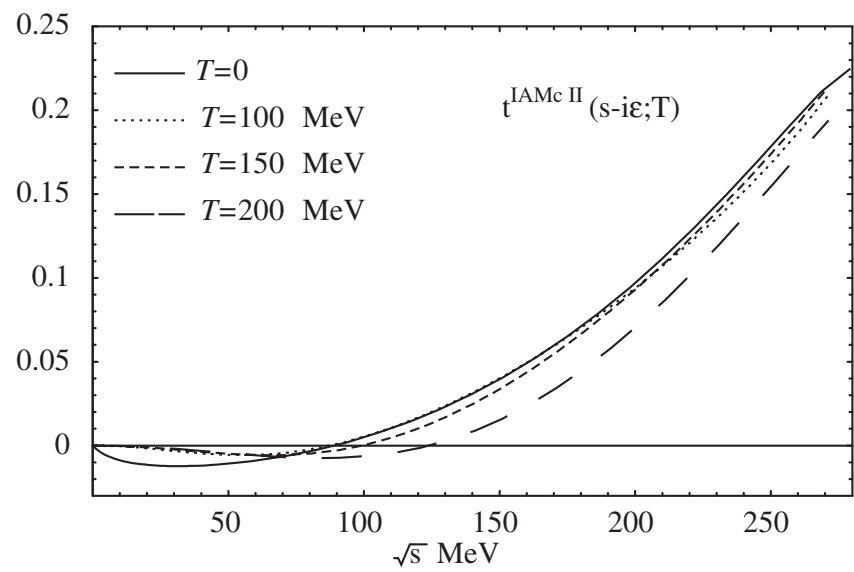

FIG. 4. Extended unitarized amplitude at finite temperature, in the first and second Riemann sheets. No real pole is observed for temperatures up to $T \simeq 200 \mathrm{MeV}$. 
octet $[23,24,36]$. Thus, these results support the existence of such a scalar nonet, in agreement with models where those states are included explicitly. However, there is an important discrepancy about their $\bar{q} q$ nature. In fact, a recent analysis of the large- $N_{c}$ behavior of the low-lying resonances generated by unitarized $\mathrm{ChPT}$ [36] shows that poles of the members of the scalar nonet, such as the $f_{0}(600)$, do not behave as a pure $\bar{q} q$ state unlike for instance the $\rho$ or the other members of the vector meson octet. The observed $f_{0}(600)$ state main content could then be of non- $\bar{q} q$ states with the same quantum numbers, such as tetraquark (or two-meson) or glueball states [36,37].

Therefore, when medium effects are included in models where the $\sigma$ is explicitly introduced [3-5,11,12], it is expected that $m_{\sigma} \sim\langle\sigma\rangle$, the order parameter, as temperature or density is increased [12]. In that framework, it is not surprising that near the phase transition, the $\sigma$ becomes degenerate with the pion, both in mass and width, since in the chiral limit the only source for the $\sigma$ mass is its vacuum expectation value so that the complex $\sigma$ pole approaches the real axis. However, as we have commented above, the observed $f_{0}(600)$ might not have such a simple structure, and this should show up in the pole behavior as the system approaches the chiral phase transition, although bearing in mind the limitations of our approach as $T$ approaches $T_{c}$.

Our unitarized ChPT results show that the $f_{0}(600)$ exhibits a clear tendency to reduce the mass of the pole as the temperature increases. For $T$ greater than about $100 \mathrm{MeV}$, this effect makes also the width decrease, overcoming the width increase due to thermal phase space. This could be interpreted as a tendency of this state to become the singlet state partner of the pion in the limit of chiral symmetry restoration. However, the size of the thermal width when the chiral transition has already been reached indicates that this state is not degenerated with the pion. Thus, although a state with the numbers of the $\bar{q} q$ isospin singlet survives near the critical point, it does not seem to behave like a $\bar{q} q$ state at finite temperature.

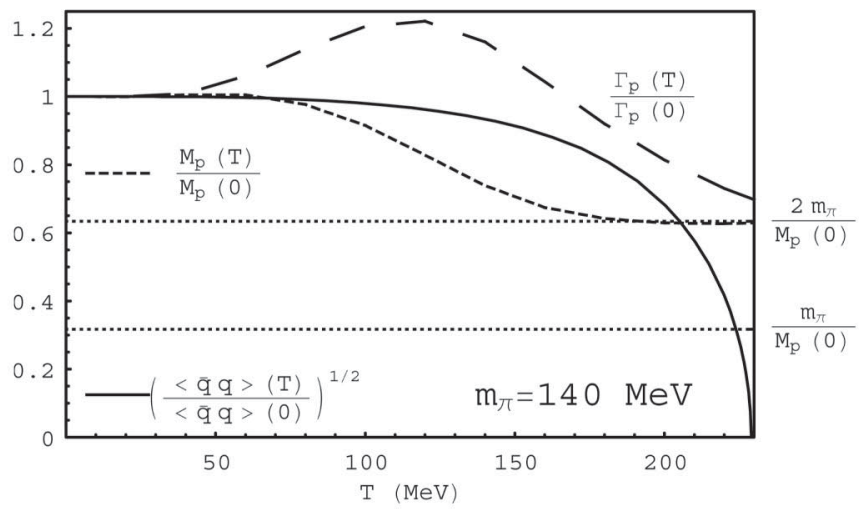

The situation is summarized in Fig. 5, where we compare the pole mass $M_{p}(T)$ with $\langle\bar{q} q\rangle^{1 / 2}$. The reason for doing so is that $\langle\sigma\rangle$ in the $O(4)$ model corresponds to $f_{\pi}$ to leading order. On the other hand, from the Gell-MannOakes Renner (GOR) relation $f_{\pi}^{2}=-m_{q}\langle\bar{q} q\rangle / m_{\pi}^{2}$ [38] where $m_{q}=m_{u}=m_{d}$, we expect $f_{\pi}$ to scale with $T$ like $\langle\bar{q} q\rangle^{1 / 2}$ if the $T$-dependence of $m_{\pi}$ is ignored. This is a good approximation in one-loop ChPT [18] whereas for two-loops there are pion mass corrections to the GOR relation when it is expressed in terms of $T$-dependent quantities [39]. We have taken the quark condensate from the virial expansion [20], where the pressure and the quark condensate are expressed in terms of $T=0$ scattering amplitudes. The results shown in Fig. 5 are obtained with the $\mathcal{O}\left(p^{4}\right)$ pion scattering amplitudes, giving a critical temperature $T_{c} \simeq 230 \mathrm{MeV}$, similarly to the ChPT perturbative three-loop calculation in [19] for the pion gas. The differences introduced by considering unitarized amplitudes are small in the virial approach [20].

In Fig. 5 we also show the results for $m_{\pi}=10 \mathrm{MeV}$, i.e., close to the chiral limit. Here, a technical remark is in order. As commented above, we are expressing our amplitudes in terms of the scale-independent low-energy constants $\bar{l}_{i}$, with the same convention as in [27]. Namely, they are related to the one-loop renormalized constants as $l_{i}^{R}(\mu)=\gamma_{i}\left[\bar{l}_{i}+\log \left(m_{\pi}^{2} / \mu^{2}\right)\right] /\left(32 \pi^{2}\right)$ where $\gamma_{i}$ are numerical factors and $\mu$ is the renormalization scale. Since the $l_{i}^{R}(\mu)$ are mass-independent, when we change the pion mass from $m_{1}$ to $m_{2}$, we should also change $\bar{l}_{i} \rightarrow$ $\bar{l}_{i}+\log \left(m_{1}^{2} / m_{2}^{2}\right)$ in the amplitudes when calculating the poles. The same observation applies to the numerical value of $f_{\pi}$ we are using, which is also mass-dependent since to one loop, $f_{\pi}=f\left[1+\bar{l}_{4} m_{\pi}^{2} /\left(16 \pi^{2} f^{2}\right)\right]$ [27], where $f \simeq$ 87.6 MeV is the pion decay constant in the chiral limit.

For the physical pion mass, the mass curve departs from the condensate one as it approaches $2 m_{\pi}$. This is consistent with the idea that when the $\bar{q} q$ mean value is negligible, the non- $\bar{q} q$ component of the $f_{0}(600)$ state still survives in the

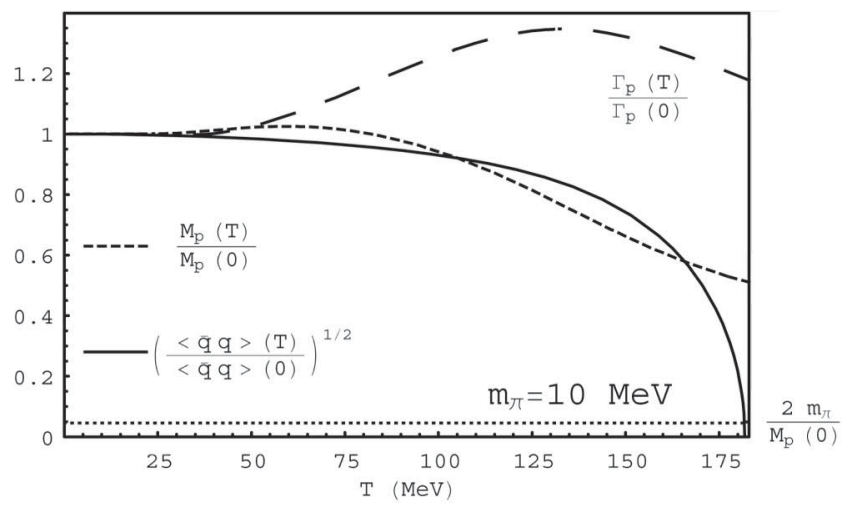

FIG. 5. Comparison between the $f_{0}(600)$ pole relative mass and width with the quark condensate, for the physical pion mass (left) and near the chiral limit, for $m_{\pi}=10 \mathrm{MeV}$ (right). 
thermal bath as a short-lived state. The departure from the $\sigma$-like evolution is even greater if effects beyond one loop are considered. For instance, $m_{\pi}(T)$ calculated to first order in the pion density [40] with the IAM $T=0$ partial waves, decreases considerably near the chiral transition so that it gets even further from $M_{p}(T)$, while $f_{\pi}(T)$ at twoloops starts increasing from $T>150 \mathrm{MeV}$ [39].

The above conclusions are confirmed when reducing the pion mass, so that explicit chiral symmetry breaking terms are negligible, chiral symmetry restoration takes place at a lower temperature (for $m_{\pi}=10 \mathrm{MeV}$ we get $T_{c} \simeq$ $182 \mathrm{MeV}$, also close to the chiral limit three-loop result in [19]) and the $\sigma$-like behavior for the pole should be more visible. The results shown in Fig. 5 for $m_{\pi}=$ $10 \mathrm{MeV}$ are qualitatively not very different from the physical mass case. The values of the mass and width of the pole at $T=0$ are now $M_{p} \simeq 406.2 \mathrm{MeV}$ and $\Gamma_{p} \simeq 522.7 \mathrm{MeV}$. Therefore, the pion mass reduction produces a larger effect in the width (due to the increase of phase space) than in the mass, consistently with the idea that the main contribution to the $f_{0}(600)$ mass comes from the spontaneous chiral symmetry breaking scale and not from the explicit part. The larger width makes the interpretation of the $f_{0}(600)$ in terms of a real particle still more doubtful. At finite $T$, the width increases again first by thermal phase space and decreases near the transition along with the pole mass, which decreases monotonically. We see that the mass curve is now closer to the condensate than for the physical pion mass, which confirms our previous comment about restoration in the chiral limit. However, the width remains again very large at the critical point and in fact the mass is even far from $2 m_{\pi}$, revealing once more the possible presence of non- $\bar{q} q$ components. The GOR deviations and pion mass $T$-dependence are negligible near the chiral limit $[39,40]$.

These results, combined with the absence of real poles for the extended amplitudes discussed in Sec. IV, which we have also checked for $m_{\pi}=10 \mathrm{MeV}$, lead us to conclude that in our finite $T$ approach there is no $f_{0}(600)-\pi$ degeneracy at the critical point.

Finally, we comment on the implications of our results for the finite temperature nature of the $\rho$ resonance. As discussed in Sec. III, our main result is that near the critical point the $\rho$ exhibits a substantial thermal broadening, which increases with temperature. The mass reduction is small, of only about $50 \mathrm{MeV}$ at $T=200 \mathrm{MeV}$. This behavior is compatible with other model calculations [41] and its implications for the pion electromagnetic form factor [42], which is the quantity entering directly into the dilepton rate, are consistent with recent experimental data on the CERN-SPS dilepton spectrum $[43,44]$ and on the $\rho$ shape from the $\pi^{+} \pi^{-}$spectrum in RHIC-STAR [45]. On the other hand, the original Brown-Rho scaling hypothesis [46] predicts that the $\rho$ mass should decrease with medium effects roughly as the condensate, i.e., $M_{T} / M_{0} \simeq$ $\langle\bar{q} q\rangle_{T} /\langle\bar{q} q\rangle_{0}$, at least close to the transition point. This hypothesis has been supported by approaches based upon the hidden local symmetry framework [47], where the $\rho$ is introduced as a gauge field of the local chiral symmetry, and implies that at the critical temperature the $\rho$ should become degenerate with the pion in the chiral limit. The BR dropping-mass scenario predictions [48] are compatible with the CERES dilepton data [43] but they seem to be in conflict with the recent NA60 dimuon data [44,49]. The latter has been nevertheless questioned in $[47,50]$, where, among other arguments like the violation of vector meson dominance, it is claimed that the $\mathrm{BR} M_{\rho}$ dropping takes really place only near $T_{c}$, whereas the mass changes very little until about $T \simeq 125 \mathrm{MeV}$.

We present in Fig. 6 our results for the $\rho$ mass compared to the quark condensate, where we have extrapolated our IAM poles shown in Fig. 1 to the temperature $T_{0} \simeq$ $310 \mathrm{MeV}$ where the pole mass would vanish. Note that $T_{0}$ is notably far from the chiral transition point estimated by the vanishing of the chiral condensate at $T_{c} \simeq$ $230 \mathrm{MeV}$, calculated in the virial approach as commented previously. We have also represented a fit of our results with a curve of the type $M_{\rho}(T) / M_{\rho}(0)=\left[1-\left(T / T_{0}\right)^{n}\right]^{\alpha}$ with $n$ integer, as suggested in [49]. We get a good agreement for $n=6$ and $\alpha \simeq 0.8$. Therefore, although our results are compatible with a dropping-mass behavior with only sizable mass decrease very near $T_{0}$ [47,50], the value of such "critical" temperature is rather high and the mass curve does not follow the quark condensate. On the other hand, as commented above, our main effect is the $\rho$ broadening, whereas in [47] the width does not change much except near the critical point where it also drops. In addition to the thermal phase space increasing, this is related to the behavior of the $\rho \pi \pi$ effective vertex which also drops in [47], whereas in our case grows with $T$ [26], making the width grow even more. In conclusion, unitarized ChPT is not fully incompatible with the BR scenario in what con-

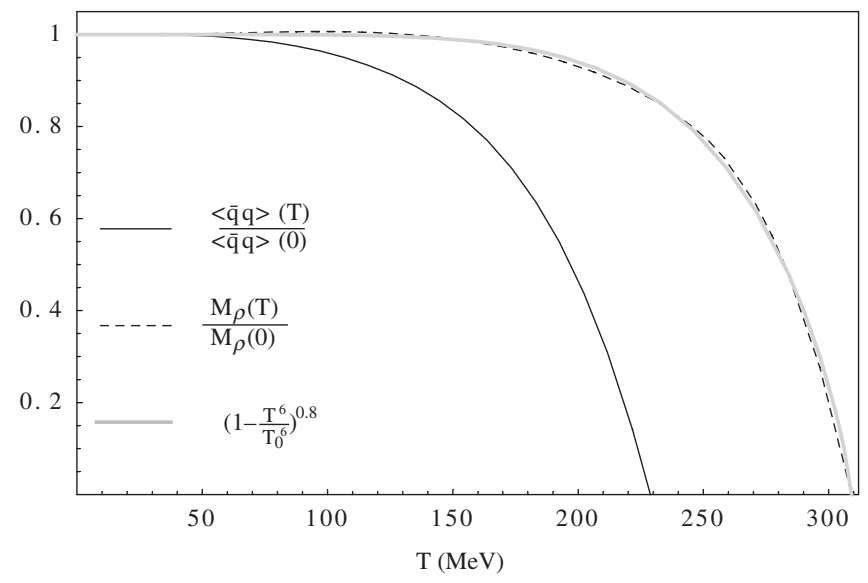

FIG. 6. Comparison between the $\rho$ pole and the chiral condensate. Here, we include a simple fit of the form suggested by dropping-mass scenarios, where $T_{0} \simeq 310 \mathrm{MeV}$ is the temperature at which the extrapolated $\rho$ mass vanishes. 
cerns the qualitative behavior of the $\rho$ mass, but the nature of the $\rho$ state when the system approaches the chiral transition is very different in both approaches: we find a wide state of relatively little change in mass, while in BRlike approaches the $\rho$ tends to a massless narrow state. In other words, the thermal behavior of the $\rho$ in our approach is dominated by nonchiral restoring effects, unlike in the BR scenario. We also point out that, due to the vector nature of the $\rho$ resonance, it is not obvious that one can relate its chiral restoring behavior with its $\bar{q} q$ content, as we did in the scalar $f_{0}(600)$ case which has the vacuum quantum numbers.

\section{CHIRAL RESTORING MEDIUM EFFECTS}

In the previous sections, we have analyzed the temperature behavior of the $\pi \pi$ scattering poles in the 00 and 11 channels. One important conclusion of that analysis has been the notorious influence of thermal phase medium effects, which in the $f_{0}(600)$ case compete with chiral restoration with the result that no observable effect is produced when the real part of the pole approaches the two-pion threshold. This thermal phase space effect is the result of the scattering with pions in the thermal bath, as explained in Sec. II.

In this section we will consider the influence of other chiral restoring effects such as nuclear density. For that purpose, we will follow a simplified treatment, taking $T=$ 0 and encoding those effects in an effective decreasing $f_{\pi}$. The GOR relation is valid to linear order in nuclear density at $T=0$ [51] as long as the $f_{\pi}$ associated to the time component of the axial current is used (it is generally different from the space component in the medium [52]) and therefore, we expect $f_{\pi}^{2}$ to scale like the quark condensate in the nuclear medium. In addition, the density dependence of the pion mass is rather weak and can be neglected in a first approximation so that to leading order [51]:

$$
\frac{f_{\pi}^{2}(\rho)}{f_{\pi}^{2}(0)} \simeq \frac{\langle\bar{q} q\rangle(\rho)}{\langle\bar{q} q\rangle(0)} \simeq\left(1-\frac{\sigma_{\pi N}}{m_{\pi}^{2} f_{\pi}^{2}(0)} \rho\right) \simeq\left(1-0.35 \frac{\rho}{\rho_{0}}\right)
$$

where $\rho$ is the nuclear density, $\sigma_{\pi N} \simeq 45 \mathrm{MeV}$ [53] is the pion-nucleon sigma term and $\rho_{0} \simeq 0.17 \mathrm{fm}^{-3}$ is the normal or saturation nuclear matter density.

In this approach the interaction between pions and nuclear matter is considered in an effective mean-field way. This is the same philosophy followed in earlier linear- $\sigma$ model analysis [4,5] which introduce the linear density dependence only through the function $\Phi(\rho)=$ $\langle\sigma\rangle(\rho) /\langle\sigma\rangle(0) \sim f_{\pi}(\rho) / f_{\pi}(0)$. The same procedure of simulating medium effects for pion scattering only in the reduction of $f_{\pi}$ is followed in $[6,8]$. More elaborated finitedensity analysis of the pion scattering amplitude [7] conclude that to linear order in density the amplitude corrections roughly amount to consider the in-medium pion- propagator and pion decay constant. We do not consider corrections in the pion self-energy, but even when those are taken into account [7] the result is still a significant threshold enhancement with no in-medium broadening of the $f_{0}(600)$. The approach followed in [14] includes additional in-medium corrections and the dropping behavior of $f_{\pi}$ is automatically incorporated [54]. In that approach the pion propagator is also renormalized in the medium, other effects being subdominant, and the results obtained for threshold enhancement are similar to other approaches. All these works support the conclusion that for low nuclear density and $T=0$ there are no intermediate states producing a significant broadening as with the thermal space broadening discussed in Sec. II, which is proportional to Bose-Einstein pion distributions and is therefore absent at $T=0$. For instance, in [14], the renormalized in-medium pion propagators induce effectively new intermediate states which can be interpreted as coming from collisions with matter particles, but these new channels do not make the $f_{0}(600)$ broader [55]. Nevertheless, we stress that we do not mean to describe the full $\pi \pi$ scattering at finite density only with this approach. Our main purpose is to see whether pure chiral symmetry restoration terms as those given by (25) can reproduce typical threshold enhancement effects in our unitarized ChPT scheme.

Although we expect the reduction of $f_{\pi}$ to be the dominant effect for precursors of chiral symmetry restoration in the $I=J=0$ channel, this is not so clear for the $I=J=$ 1 channel, for which no observable threshold effects are seen in $\pi \pi$ scattering in nuclei [17]. The BR scaling suggests that the mass of the $\rho$ resonance should also drop with increasing density $[46,48,56]$ and near the critical density it should follow the condensate curve (25). Since the $\rho$ resonance is a narrow state, this implies that the width also vanishes by phase space reduction, so that the pole is driven by chiral restoration towards threshold, as the $f_{0}(600)$ pole. The crucial point, as far as finitedensity effects are concerned, is whether the threshold enhancement is visible for densities $\rho \lessgtr \rho_{0}$, which are those accessible experimentally [15-17].

The evolution of the poles with decreasing $f_{\pi}$ is shown in Fig. 7. In both channels, we have used the extended amplitude (20), since the distance of the pole to the real axis is reduced for increasing density. A salient feature is that in both channels the poles move towards the origin. This can be qualitatively understood by noting that the IAM (23) scales with $f_{\pi}$ as $t=\tilde{t}_{2}^{2} /\left(f_{\pi}^{2} \tilde{t}_{2}-\tilde{t}_{4}-\sigma \tilde{t}_{2}^{2}\right)$, where the functions with tilde are independent of $f_{\pi}$, and similarly for the extended amplitudes. The domain of convergence of the chiral expansion $\left(f_{\pi}^{2} \tilde{t}_{2} \gg \tilde{t}_{4}, \tilde{t}_{2}^{2}\right)$ around threshold is therefore reduced when decreasing $f_{\pi}$ and thus the value at which the denominator vanishes may be closer to the real axis.

The $f_{0}(600)$ pole mass reaches threshold at about $f_{\pi} \simeq$ $55 \mathrm{MeV}$, or density $\rho \simeq 1.86 \rho_{0}$ from (25). Since the phase 

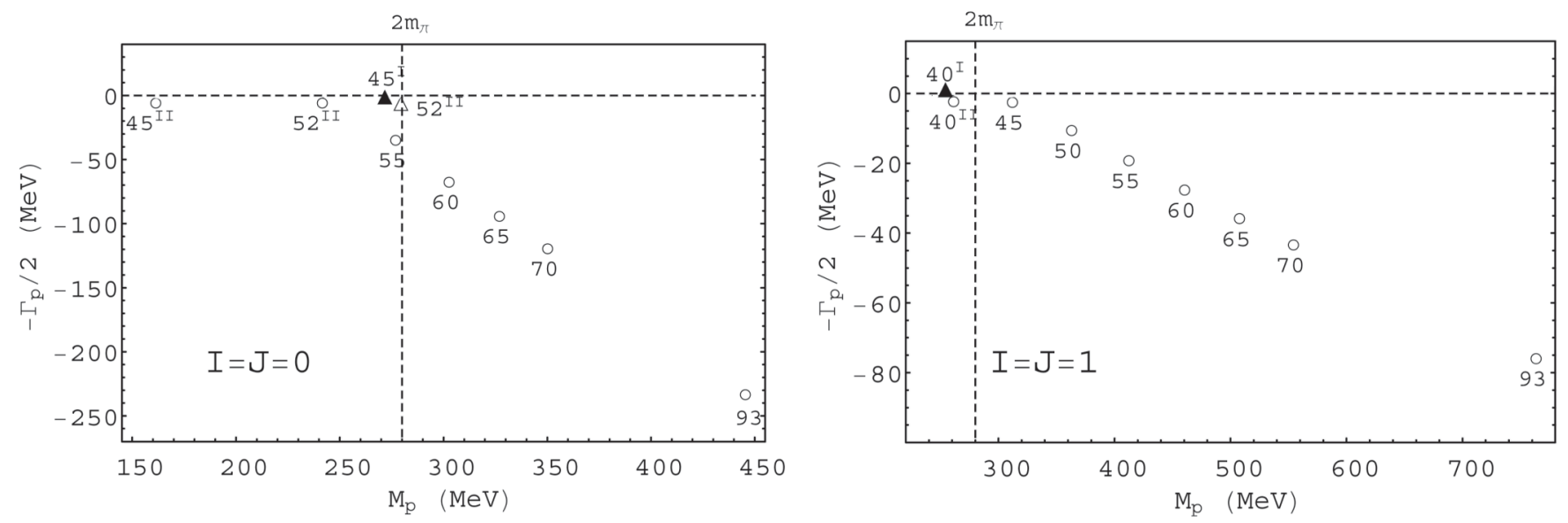

FIG. 7. Evolution of the complex poles with decreasing $f_{\pi}$ at $T=0$, corresponding to chiral restoring finite density effects through (25). Attached to every point is the value of $f_{\pi}$ in $\mathrm{MeV}$ units. All the points correspond to poles in the second Riemann sheet, except the black triangles which are in the first sheet (bound states).

space effect is absent, the resonance becomes narrow so that the mass reduction implies also a reduction in the width, which rapidly drops to zero when the real part reaches threshold. The effects on threshold enhancement are notorious as we show in Fig. 8 for $f_{\pi}=70,60$, $55 \mathrm{MeV}$ which according to (25) correspond to densities $\rho / \rho_{0}=1.2,1.7$ and 1.9, respectively. Therefore, we reproduce qualitatively the threshold enhancement at finite density obtained in previous works [4-8]. The quantitative values are not far either: in [5], the enhancement of $\left|t^{00}\right|^{2}$ at threshold for $\Phi=0.7\left(f_{\pi} \sim 78 \mathrm{MeV}\right)$ is about 9 times its vacuum value, while we get a factor of 6.5 for $f_{\pi}=$ $70 \mathrm{MeV}$. In [7], $M_{\sigma} \sim 2 m_{\pi}$ at a density $\rho \sim 2 \rho_{0}$, in good agreement with our values, while in [8] the complex pole reaches threshold at about $f_{\pi} \sim 65 \mathrm{MeV}$. The results for the pole mass and width in [55] are also similar to ours.

It is even more interesting what happens when we let the density increase further. At a given point $\left(f_{\pi} \simeq 52 \mathrm{MeV}\right)$ the pole width vanishes and there are two real poles in the second sheet, which correspond to virtual states. Remember that, according to our discussion in Sec. IV, the number of real poles in the second Riemann sheet has to be zero or an even number once we have extended properly the amplitude to remove spurious poles and provided the amplitude remains positive at threshold. When the density is increased a bit further, one of those poles jumps to the first sheet, becoming a $\pi \pi$ bound sate. This happens in the 00 channel at $f_{\pi}=45 \mathrm{MeV}$, as indicated in Fig. 7. For that value, the extended amplitude at threshold becomes negative because the $\mathcal{O}\left(f_{\pi}^{2}\right) t_{2}$ term no longer dominates and therefore the same argument of Sec. IV leads to the conclusion that there is an odd number of poles in the first and in the second sheets, one pole in this case. This pole behavior is in agreement with that found in [8], where the "pole doubling" in the second sheet is found at about $f_{\pi} \sim 60 \mathrm{MeV}$ and the movement of one of the poles to the first sheet is at $f_{\pi} \sim 50 \mathrm{MeV}$.

The evolution of the $f_{0}(600)$ pole with density in this approach is now closer to a $\sigma$-like $\bar{q} q$ term, as shown in Fig. 9. The mass follows now quite closely the curve of $f_{\pi}$ for increasing density. We are plotting the second-sheet pole, which we observe that approaches the pion mass and
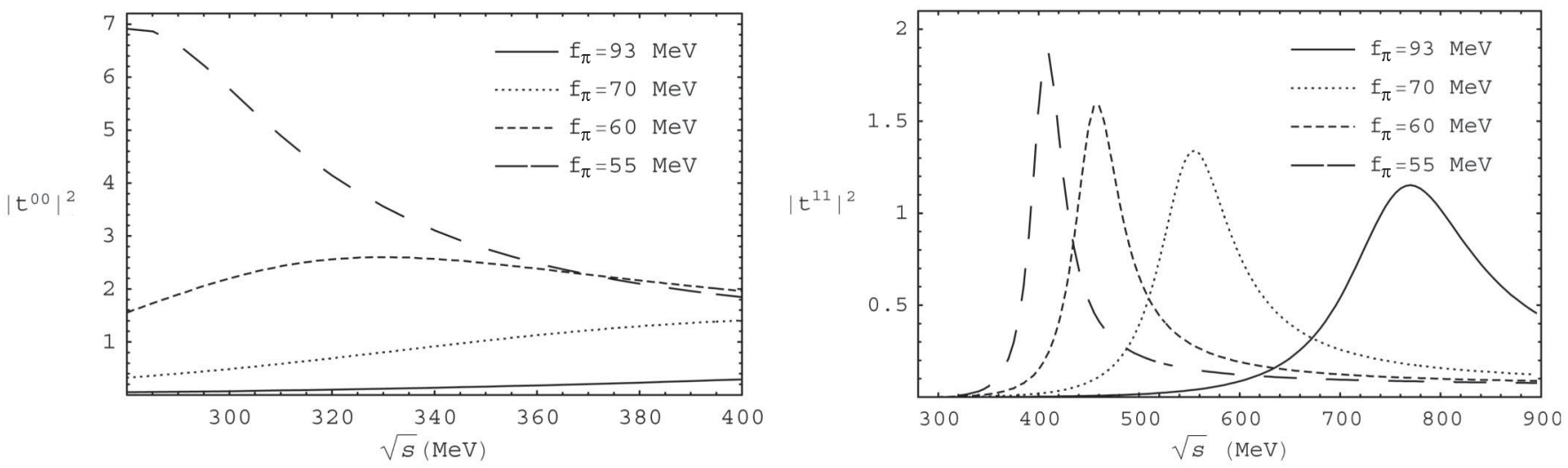

FIG. 8. Squared modulus of the $t^{00}$ and $t^{11}$ unitarized partial waves for different values of $f_{\pi}$. 

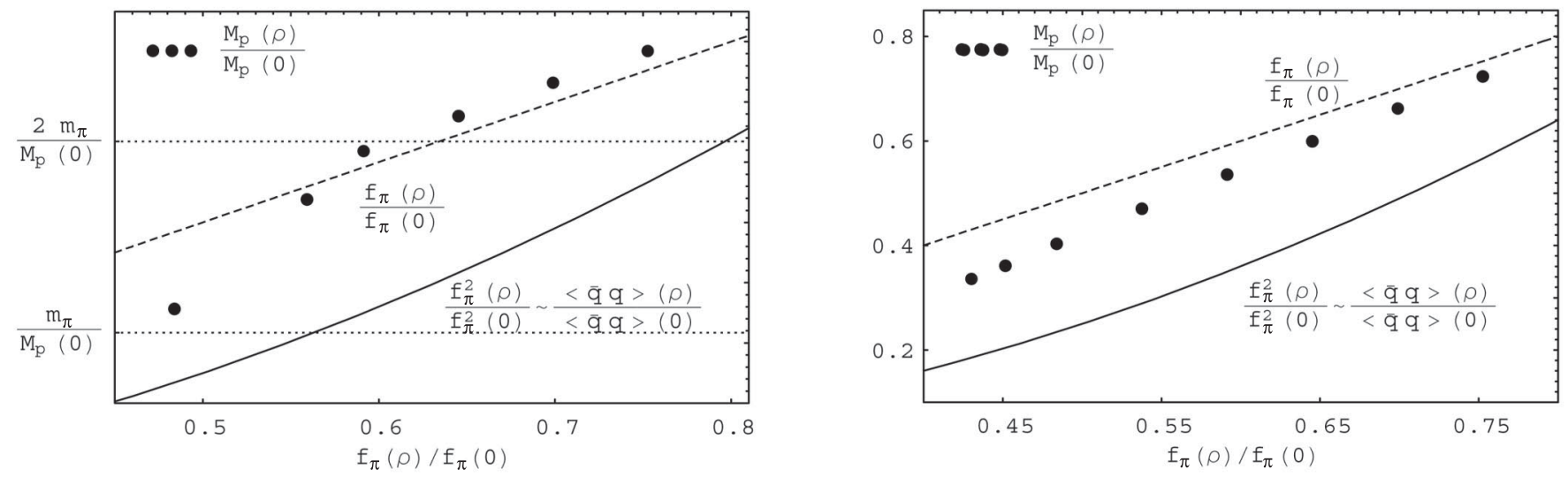

FIG. 9. Scaling with $f_{\pi}$ of the pole masses in the $I=J=0$ (left) and $I=J=1$ (right) channels. The points are the mass values of the second-sheet poles in Fig. 7, the dashed line is the linear density dependence $f_{\pi}(\rho) / f_{\pi}(0)$ and the full line is the quadratic $f_{\pi}^{2}(\rho) / f_{\pi}^{2}(0)$ which scales approximately like the quark condensate, cf., Eq. (25).

in fact it does it more rapidly than the $f_{\pi}$ curve, approaching the $f_{\pi}^{2}$ one. Our conclusion is then that at sufficiently high densities a virtual $\bar{q} q$-like state, or any other state behaving in the same way near chiral restoration, degenerates with the pion and coexists with a $\pi \pi$ bound state. Nevertheless, we should bear in mind that our approach relies on the ChPT power counting and therefore it losses validity as $f_{\pi}$ is reduced.

As for the $I=J=1$ channel, first we see that the pole is still rather away from the real axis when the $f_{0}(600)$ pole arrives threshold, which would produce a less important threshold enhancement for this channel for the relevant density range, as it can be observed in Fig. 8, consistently with the previously mentioned experimental information. When the density is further increased, there also appears a bound state joint by a virtual state in this channel, although the arguments used in Sec. IV for the $f_{0}(600)$ channel do not apply here since the perturbative amplitude vanishes at threshold. This simultaneous "softening" of the $f_{0}(600)$ and $\rho$ poles for decreasing $f_{\pi}$ has also been noted in [6].

We also see that if only these finite-density effects are considered, the $\rho$ mass follows quite closely a BR scaling pattern, as represented in Fig. 9. The mass scales with $f_{\pi}$, i.e., with $\langle\bar{q} q\rangle^{1 / 2}$ and gradually moves to the $\langle\bar{q} q\rangle$ curve. This is in agreement with recent analysis in this context [56] which show that the vector meson masses scale like $\langle\bar{q} q\rangle^{1 / 2}$ for low densities and like the condensate near the transition point. In any case, we insist that this would reflect only the finite-density effects associated with chiral restoration, which may not be the only relevant medium effects.

\section{CONCLUSIONS}

Using methods based on the unitarization of the elastic $\pi \pi$ scattering in one-loop chiral perturbation theory, we have studied the behavior of pion scattering poles with the presence of medium effects such as temperature and den- sity, as the system approaches the chiral phase transition. The unitarization framework we have employed is the inverse amplitude method. For the temperature evolution we have used thermal amplitudes, whereas we mimic chiral restoration effects associated to nuclear density by studying the variation with the pion decay constant in the linear density GOR approximation at $T=0$.

The $I=J=0$ pole in $\pi \pi$ scattering near the chiral phase transition remains a broad state with a pole position $M_{p} \simeq 2 m_{\pi}$ but nonvanishing $\Gamma_{p}$. This implies that only with finite temperature effects is very unlikely that there could be observable precursors of chiral symmetry restoration like an enhancement of the $\pi \pi$ scattering cross section, as claimed by various authors. As we have shown in detail, the reason for that is that thermal phase space increases the width considerably before chiral restoring is effective, so that near the critical point the resonance spectral function is widely spread. In fact, we have shown that our results can be qualitatively understood in terms of the generalized decay rate of a wide resonance into two pions.

At finite temperature we do not find additional poles in the second Riemann sheet near the real axis (virtual states), which is consistent with the $f_{0}(600)$ remaining a broad resonance. For the analysis of real poles, it is crucial to extend the unitarized amplitude in order to account properly for the Adler zeros. In this way, we have shown that we can get rid of nonphysical poles appearing below the $\pi \pi$ threshold in the 00 and 20 channels. The extended amplitudes are almost indistinguishable from the IAM ones in the physical region, i.e., above threshold. This part of our work is interesting also for formal reasons, since one does not lose the powerful unitarity and chiral symmetry constraints when demanding also a behavior below threshold consistent with the perturbative Adler zeros.

We have provided an interpretation of our results at finite $T$ in terms of the nondegeneracy of "chiral partners" near the critical temperature. The fact that the $f_{0}(600)$ width 
remains large at the critical point, even near the chiral limit, supports this fact and is also consistent with a sizable presence of non $\bar{q} q$ components in the thermal $f_{0}(600)$ state. This is confirmed also by the differences between the mass and the quark condensate curves as $T$ approaches the critical point. Our results for the $\rho$ state in the thermal case predict a broadening behavior with slight mass reduction and are compatible with several broadening- $\rho$ scenarios. They are in conflict however with dropping-mass descriptions, with which we only agree in the dropping behavior of the mass, but with a vanishing temperature larger than the critical temperature predicted by the quark condensate.

When the pion decay constant is reduced, to reproduce linear density chiral restoring effects at $T=0$, we obtain a sharp chiral restoration pattern in the $I=J=0$ channel, showing threshold enhancement at densities similar to those obtained in different models and qualitatively consistent with experiments, in spite of the fact that our finitedensity approach includes only a limited type of contributions. When the $f_{0}(600)$ pole reaches threshold, two poles appear on the second Riemann sheet, one of them remains a virtual state that tends to degenerate with the pion, while the other becomes a two-pion bound state. The nature of the $f_{0}(600)$ within this approach resembles much more the typical $\bar{q} q$ one of linear $\sigma$ models. As for the $\rho$, the finitedensity effects associated to chiral symmetry restoration produce a Brown-Rho like scaling of the pole mass, which moves towards threshold first as the square root of the condensate for moderate densities and as the condensate for higher ones. The $\rho$ gets narrower with only these effects and a bound-virtual state pair also appears at high densities.

In summary, the presence of chiral symmetry precursors related to real poles in the $I=J=0$ channel is disfavored in our approach when finite temperature effects are included, while pure density effects favor these precursors and Brown-Rho scaling. Therefore, in a relativistic heavy ion collision, where both temperature and density effects are relevant, our approach does not predict any measurable effect related to the pole evolution other than the $\rho$ widening, observable in dilepton data. On the other hand, our results for density-like effects are compatible with inmedium $\pi \pi$ production experiments, where threshold enhancement has been observed, despite the limitations of our approach.

As a future extension of this work, a finite temperature and density analysis of the meson-meson scattering poles in the $S U(3)$ case may also reveal important information about chiral symmetry restoration, where the presence of heavier states becomes important.

\section{ACKNOWLEDGMENTS}

We are grateful to J.R. Peláez, G. Ríos and R. García Martín for very useful comments and discussions. We also acknowledge financial support from the Spanish research projects FPA2004-02602, FPA2005-02327, PR27/0513955-BSCH, UCM-CAM 910309 and from the F.P.I. programme (BES-2005-6726).
[1] W. M. Yao et al. (Particle Data Group), J. Phys. G 33, 1 (2006).

[2] T. Hatsuda and T. Kunihiro, Phys. Rev. Lett. 55, 158 (1985).

[3] S. Chiku and T. Hatsuda, Phys. Rev. D 57, R6 (1998).

[4] T. Hatsuda, T. Kunihiro, and H. Shimizu, Phys. Rev. Lett. 82, 2840 (1999).

[5] D. Jido, T. Hatsuda, and T. Kunihiro, Phys. Rev. D 63, 011901(R) (2000).

[6] K. Yokokawa, T. Hatsuda, A. Hayashigaki, and T. Kunihiro, Phys. Rev. C 66, 022201(R) (2002).

[7] D. Davesne, Y. J. Zhang, and G. Chanfray, Phys. Rev. C 62, 024604 (2000).

[8] A. Patkos, Z. Szep, and P. Szepfalusy, Phys. Rev. D 66, 116004 (2002).

[9] P. Schuck, W. Norenberg, and G. Chanfray, Z. Phys. A 330, 119 (1988).

[10] A. Patkos, Z. Szep, and P. Szepfalusy, Phys. Rev. D 68, 047701 (2003).

[11] Y. Hidaka, O. Morimatsu, T. Nishikawa, and M. Ohtani, Phys. Rev. D 68, 111901(R) (2003).
[12] Y. Hidaka, O. Morimatsu, T. Nishikawa, and M. Ohtani, Phys. Rev. D 70, 076001 (2004).

[13] L. Roca, E. Oset, and M. J. Vicente Vacas, Phys. Lett. B 541, 77 (2002).

[14] H. C. Chiang, E. Oset, and M. J. Vicente-Vacas, Nucl. Phys. A644, 77 (1998); D. Cabrera, E. Oset, and M. J. Vicente Vacas, Phys. Rev. C 72, 025207 (2005).

[15] F. Bonutti et al. (CHAOS collaboration), Nucl. Phys. A 677, 213 (2000).

[16] A. Starostin et al. (Crystal Ball Collaboration), Phys. Rev. Lett. 85, 5539 (2000).

[17] J. G. Messchendorp et al., Phys. Rev. Lett. 89, 222302 (2002).

[18] J. Gasser and H. Leutwyler, Phys. Lett. B 184, 83 (1987).

[19] P. Gerber and H. Leutwyler, Nucl. Phys. B321, 387 (1989).

[20] A. Dobado and J. R. Peláez, Phys. Rev. D 59, 034004 (1998); J. R. Peláez, Phys. Rev. D 66, 096007 (2002).

[21] T. N. Truong, Phys. Rev. Lett. 61, 2526 (1988); 67, 2260 (1991); A. Dobado, M. J. Herrero, and T. N. Truong, Phys. Lett. B 235, 134 (1990). 
[22] A. Dobado and J. R. Peláez, Phys. Rev. D 47, 4883 (1993); 56, 3057 (1997).

[23] J. A. Oller, E. Oset, and J. R. Peláez, Phys. Rev. Lett. 80, 3452 (1998).

[24] A. Gómez Nicola and J. R. Peláez, Phys. Rev. D 65, 054009 (2002).

[25] A. Gómez Nicola, F. J. Llanes-Estrada, and J. R. Peláez, Phys. Lett. B 550, 55 (2002).

[26] A. Dobado, A. Gómez Nicola, F. J. Llanes-Estrada, and J. R. Peláez, Phys. Rev. C 66, 055201 (2002).

[27] J. Gasser and H. Leutwyler, Ann. Phys. (N.Y.) 158, 142 (1984).

[28] S. Weinberg, Phys. Rev. Lett. 17, 616 (1966).

[29] H. A. Weldon, Phys. Rev. D 28, 2007 (1983); Ann. Phys. (N.Y.) 214, 152 (1992).

[30] N. Kaiser, Phys. Rev. C 59, 2945 (1999).

[31] C. Amsler and N. A. Tornqvist, Phys. Rep. 389, 61 (2004).

[32] H. A. Weldon, Ann. Phys. (N.Y.) 228, 43 (1993).

[33] D. V. Bugg, Phys. Lett. B 572, 1 (2003).

[34] A. Gómez Nicola, J. R. Peláez, and G. Rios (unpublished).

[35] R. Delbourgo and M. D. Scadron, Mod. Phys. Lett. A 10, 251 (1995).

[36] J. R. Peláez, Phys. Rev. Lett. 92, 102001 (2004); Mod. Phys. Lett. A 19, 2879 (2004).

[37] R. L. Jaffe, arXiv:hep-ph/0701038.

[38] M. Gell-Mann, R. J. Oakes, and B. Renner, Phys. Rev. 175, 2195 (1968).

[39] D. Toublan, Phys. Rev. D 56, 5629 (1997).

[40] A. Schenk, Phys. Rev. D 47, 5138 (1993).

[41] R. D. Pisarski, Phys. Rev. D 52, R3773 (1995); V. Koch and C. Song, Phys. Rev. C 54, 1903 (1996); C. Song and V.
Koch, Phys. Rev. C 54, 3218 (1996); R. Rapp and J. Wambach, Eur. Phys. J. A 6, 415 (1999); Adv. Nucl. Phys. 25, 1 (2002); H.-J. Schulze and D. Blaschke, Phys. Lett. B 386, 429 (1996); Phys. Part. Nucl. Lett. 1, 70 (2004); H. van Hees and R. Rapp, Phys. Rev. Lett. 97, 102301 (2006).

[42] A. Gómez Nicola, F. J. LLanes-Estrada, and J. R. Peláez, Phys. Lett. B 606, 351 (2005).

[43] G. Agakichiev et al. (CERES Collaboration), Eur. Phys. J. C 41, 475 (2005).

[44] R. Arnaldi et al. (NA60 Collaboration), Phys. Rev. Lett. 96, 162302 (2006).

[45] J. Adams et al. (STAR Collaboration), Phys. Rev. Lett. 92, 092301 (2004).

[46] G. E. Brown and M. Rho, Phys. Rev. Lett. 66, 2720 (1991).

[47] M. Harada and C. Sasaki, Phys. Rev. D 74, 114006 (2006).

[48] G. E. Brown and M. Rho, Phys. Rep. 363, 85 (2002).

[49] H. van Hees and R. Rapp, arXiv:hep-ph/0604269.

[50] G.E. Brown and M. Rho, arXiv:nucl-th/0509001; arXiv:nucl-th/0509002.

[51] V. Thorsson and A. Wirzba, Nucl. Phys. A589, 633 (1995); U. G. Meissner, J. A. Oller, and A. Wirzba, Ann. Phys. (N.Y.) 297, 27 (2002).

[52] R. D. Pisarski and M. Tytgat, Phys. Rev. D 54, R2989 (1996).

[53] J. Gasser, H. Leutwyler, and M.E. Sainio, Phys. Lett. B 253, 252 (1991).

[54] C. Garcia-Recio, J. Nieves, and E. Oset, Phys. Lett. B 541, 64 (2002).

[55] M. J. Vicente Vacas and E. Oset, arXiv:nucl-th/0204055.

[56] G. E. Brown and M. Rho, Phys. Rep. 396, 1 (2004). 